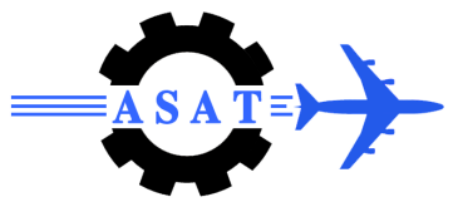

\title{
Improved Hybrid Kalman Filter for In-Flight Aircraft Engine Performance Estimation
}

\author{
LU Jun ${ }^{*}$, GUO Yingqing ${ }^{\dagger}$, ZHANG shugang ${ }^{\ddagger}$
}

\begin{abstract}
This paper proposes an improved hybrid Kalman filter(IHKF) for in-flight aircraft engine performance estimation. It's a special hybrid structure of nonlinear on-board engine model(NOBEM) and piecewise linear Kalman filter(PWKF). The outputs of NOBEM is regarded as the baseline of PWKF, while its performance deterioration factors(PDF) regarded as the augmented state vector of PWKF is on-line estimated by the deviation of measured outputs, and fed back to NOBEM which can be on-line updated next time. In addition, the finite state machine logic of work mode has been established, which can make the IHKF work better. By this approach applied to a turbofan engine, a series of simulation results show that the model can estimate the real engine performance effectively in the whole flight envelope, different engine states and severe performance deterioration condition, which lays the foundation for intelligent engine control(IEC), performance seeking control(PSC) and inflight fault detection, isolation and accommodation (FDIA).
\end{abstract}

Keywords: in-flight aircraft engine performance estimation; hybrid Kalman filter; piecewise linear Kalman filter

\section{Nomenclature}

$\begin{array}{llll}\text { LPT } & \text { Low pressure turbine } & D_{E H} & \text { Compressor efficiency } \\ \text { HPT } & \text { High pressure turbine } & D_{W L} & \text { Fan flow capacity } \\ \text { PLA } & \text { Power level angle } & D_{W H} & \text { Compressor flow capacity } \\ W_{F M} & \text { Fuel flow to main burner } & T_{1} & \text { Engine inlet temperature } \\ N_{L} & \text { Fan speed } & P_{1} & \text { Engine inlet pressure } \\ N_{H} & \text { Compressor speed } & H & \text { Altitude of aircraft } \\ T_{25} & \text { Compressor inlet temperature } & M a & \text { Mach number of aircraft } \\ T_{6} & \text { LPT exit temperature } & \boldsymbol{x} & \text { State variable vector } \\ P_{31} & \text { Compressor exit pressure } & h & \text { Performance deterioration factor vector } \\ P_{6} & \text { LPT exit pressure } & u & \text { Control input vector }\end{array}$

\footnotetext{
* School of Power and Energy, Northwestern Polytechnical University, Xi'an 710072, China, npu_huoye@mail.nwpu.edu.cn

$\dagger$ School of Power and Energy, Northwestern Polytechnical University, Xi'an 710072, China, yqguo@nwpu.edu.cn

* School of Power and Energy, Northwestern Polytechnical University, Xi'an 710072, China, zsg2008100335@mail.nwpu.edu.cn
} 


$\begin{array}{llll}F_{N} & \text { Net thrust } & y_{m} & \text { Measured outputs vector } \\ S_{M F} & \text { Surge amplitude } & y_{u} & \text { Unmeasured outputs vector } \\ W_{21} & \text { Fan air flow } & z & \text { Flight condition } \\ T_{41} & \text { HPT inlet temperature } & \boldsymbol{v} & \text { System noise vector } \\ D_{E L} & \text { Fan efficiency } & \boldsymbol{w} & \text { Measurement noise vector }\end{array}$

\section{Introduction}

In-flight aircraft engine performance estimation is one of the key techniques for intelligent engine control(IEC), performance seeking control(PSC) and in-flight fault detection, isolation and accommodation (FDIA), whose reliability and precision depend on its effectiveness. In general, the engine model for performance estimation is built at normal state, but the uncertainty such as actual engine manufacturing, installation tolerances and performance deterioration in life cycle makes it working at abnormal state, which results in obvious residuals between them and can't meet the needs of practical applications.

Aimed at this problem, it mainly includes linear Kalman filter[1-10] and its extended approach[11-13]. Among those, N. Sugiyama[11] proposed a constant gain extended Kalman filter(CGEKF) composed of a constant gain and nonlinear on-board engine model (NOBEM), which not only solves the deviation of steady-state basic model, but also deals with some nonlinear characteristics of aircraft engine. And this approach has been evaluated in a single spool turbojet engine[14]. However, the numerical stability of the CGEKF may not be as robust as that of the piecewise linear Kalman filter(PWKF). Since the NOBEM receives feedback signals residuals (multiplied by a Kalman gain matrix), large residuals may drive the NOBEM out of the range that the model was designed for. Therefore, Takahisa Kobayashi ${ }^{[12]}$ proposed a hybrid Kalman filter composed of NOBEM and PWKF, where the PWKF estimates engine performance and the NOBEM regarded as the baseline runs in parallel without receiving any feedback signals from PWKF. In addition, the NOBEM is off-line updated periodically by trend monitoring systems using post-flight data.

Although the off-line updated of this approach is practical and simple relatively, it also needs a lot of communications between on-board devices and ground devices and some associated facilities. Moreover, it can work effectively under a hypothesis that the NOBEM operates in the vicinity of the degraded engine. However, this hypothesis is not suitable when it happen severe foreign object damage(FOD). Therefore, this paper proposes an IHKF based on it, and applied to a turbofan engine for validation. For simplify, the former approach is called traditional hybrid Kalman filter(THKF).

\section{IHKF}

\subsection{Model Structure}

The IHKF is composed of NOBEM and PWKF. The former belongs to the aerothermodynamic component-level model with PDF and meets the capability of on-board realtime computation, while the latter is composed of state variable model(SVM) of many steadystate points in the whole flight envelope and associated Kalman filter gain matrixes. This hybrid structure is, as shown in Fig.1, the outputs of NOBEM is regarded as the baseline of PWKF, while its PDF regarded as the augmented state vector of PWKF is on-line estimated by the deviation of measured outputs, and fed back to NOBEM which can be on-line updated next time. 
This model includes two controllable closed loops, in which the big one is composed of NOBEM and PWKF while the small one is the inside structure of PWKF. In addition, it includes two groups of outputs, NOBEM and PWKF, but the effective outputs of IHKF need to be decided by work modes.

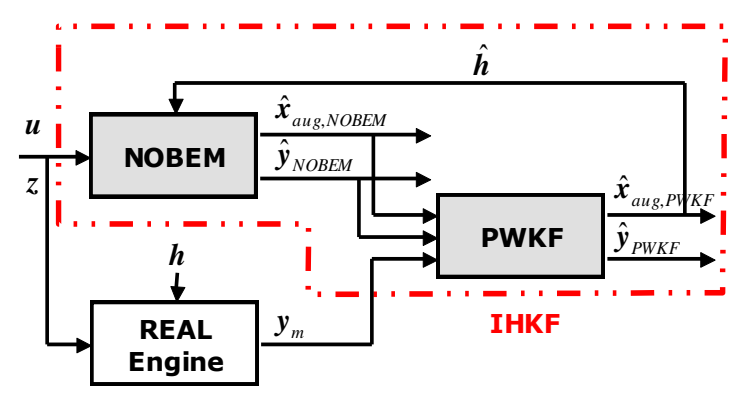

Fig. 1 The structure of IHKF

\subsection{Work Mode}

Due to two controllable closed loops and two groups of outputs, this model should be configured to the most effective work mode based on the current engine state, as shown in Tab.1. Among those, the steady-state mode is both of outputs are effective while two models update PDF simultaneously at the engine steady-state condition. The transition-state mode is the outputs of NOBEM is effective while two models freeze PDF simultaneously at the engine transition-state condition such as acceleration/deceleration, augmentation turn-on /off, etc. The abnormal mode is the outputs of PWKF are effective while two models freeze PDF simultaneously when the numerical stability of NOBEM happens. Obviously, this mode is the same as THKF, but the actual outputs are different because of different update period for PDF.

According to the change of engine state, the finite state machine logic of these modes has been established, as shown in Fig.2.

Table 1 Work Modes

\begin{tabular}{c|c|c|c|c}
\hline No. & Work mode & A & B & Effective outputs \\
\hline \hline 1 & Steady-state & $\bullet$ & $\bullet$ & A/B \\
\hline 2 & Transition-state & $\circ$ & $\circ$ & $\mathrm{A}$ \\
\hline 3 & Abnormal & $\circ$ & $\circ$ & $\mathrm{B}$ \\
\hline \hline
\end{tabular}

A- NOBEM

B- PWKF

- Update PDF

o- Freeze PDF

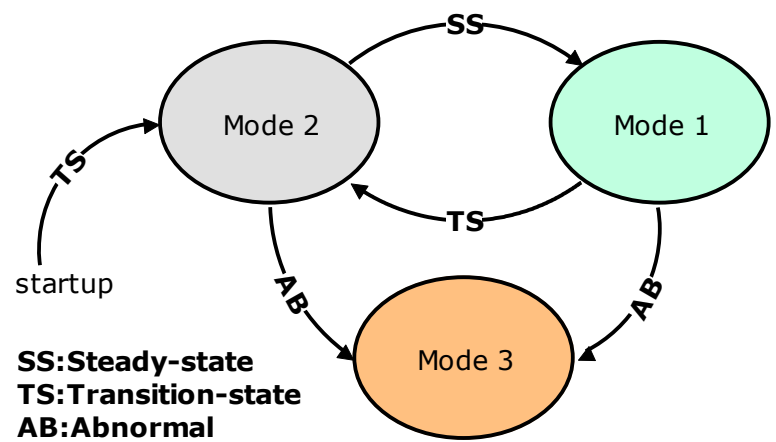

Fig. 2 The finite state machine logic of work modes 


\subsection{Model Design}

The whole processes of model design are as follow:

1) Providing the aero-thermodynamic component-level model as NOBEM

2) Defining a plurality of steady-state points in the whole flight envelop

3) Building SVM and Kalman gain matrix at each point and combining them to Kalman filter

4) Transforming Kalman filter to the sea-level condition by corrected principle and modifying the abnormal values

5) Defining the scheduled parameters and uniting all points to PWKF

6) Combining NOBEM and PWKF against the structure as shown in Fig.1

Due to the limited space, the next part only describes some of key techniques in detail.

\subsubsection{IHKF}

Suppose we has obtained an aero-thermodynamic component-level model for an aircraft engine, the represent form is

$$
\left\{\begin{array}{l}
\dot{\boldsymbol{x}}=f(\boldsymbol{x}, \boldsymbol{h}, \boldsymbol{u}, \boldsymbol{z}) \\
\boldsymbol{y}_{m}=g_{m}(\boldsymbol{x}, \boldsymbol{h}, \boldsymbol{u}, \boldsymbol{z}) \\
\boldsymbol{y}_{u}=g_{u}(\boldsymbol{x}, \boldsymbol{h}, \boldsymbol{u}, \boldsymbol{z})
\end{array}\right.
$$

At a performance baseline $\left(\boldsymbol{x}_{s s}, \boldsymbol{u}_{s s}, \boldsymbol{y}_{s s}, \boldsymbol{h}_{r e f}\right), \mathrm{SVM}$ by the linearization of eq.(1) is as follow,

$$
\left\{\begin{array}{l}
\Delta \dot{\boldsymbol{x}}=A \Delta \boldsymbol{x}+B \Delta \boldsymbol{u}+L \Delta \boldsymbol{h} \\
\Delta \boldsymbol{y}_{m}=C_{m} \Delta \boldsymbol{x}+D_{m} \Delta \boldsymbol{u}+M_{m} \Delta \boldsymbol{h} \\
\Delta \boldsymbol{y}_{u}=C_{u} \Delta \boldsymbol{x}+D_{u} \Delta \boldsymbol{u}+M_{u} \Delta \boldsymbol{h}
\end{array}\right.
$$

where $\Delta \boldsymbol{x}=\boldsymbol{x}-\boldsymbol{x}_{s s}, \Delta \boldsymbol{u}=\boldsymbol{u}-\boldsymbol{u}_{s s}, \quad \Delta \boldsymbol{y}=\boldsymbol{y}-\boldsymbol{y}_{s s}, \quad \Delta \boldsymbol{h}=\boldsymbol{h}-\boldsymbol{h}_{r e f}$, and $A, \quad B, L, \quad C_{m}, D_{m}$ , $M_{m}, C_{u}, D_{u}, M_{u}$ are system matrixs which can be obtained by partial derivative method[15] or fitting method[16].

For the real aircraft engine, the characteristics of system noise and measurement noise in nature should be considered, so eq.(2) can be transformed into

$$
\left\{\begin{array}{l}
\Delta \dot{\boldsymbol{x}}=A \Delta \boldsymbol{x}+B \Delta \boldsymbol{u}+L \Delta \boldsymbol{h}+\boldsymbol{v} \\
\Delta \boldsymbol{y}_{m}=C_{m} \Delta \boldsymbol{x}+D_{m} \Delta \boldsymbol{u}+M_{m} \Delta \boldsymbol{h}+\boldsymbol{w} \\
\Delta \boldsymbol{y}_{u}=C_{u} \Delta \boldsymbol{x}+D_{u} \Delta \boldsymbol{u}+M_{u} \Delta \boldsymbol{h}
\end{array}\right.
$$

where the covariance matrix $\boldsymbol{Q}, \boldsymbol{R}$ should be defined by rich engineering experience. Here, we assume $\boldsymbol{v}, \boldsymbol{w}$ are uncorrelated zero-mean white noise and $\boldsymbol{Q}, \boldsymbol{R}$ are diagonal matrix, $E\left(\boldsymbol{v} v^{T}\right)=Q ; E\left(w w^{T}\right)=R ; E(v)=0 ; E(w)=0$

In order to estimate $\Delta \boldsymbol{h}$ via $\Delta \boldsymbol{y}_{m}, \mathrm{SVM}$ in Kalman filter should be augmented,

$$
\left\{\begin{array}{l}
\Delta \dot{\boldsymbol{x}}_{\text {aug }}=A_{\text {aug }} \Delta \boldsymbol{x}_{\text {aug }}+B_{\text {aug }} \Delta \boldsymbol{u}+\boldsymbol{v} \\
\Delta \boldsymbol{y}_{m}=C_{\text {aug }, m} \Delta \boldsymbol{x}_{\text {aug }}+D_{\text {aug }, m} \Delta \boldsymbol{u}+\boldsymbol{w} \\
\Delta \boldsymbol{y}_{u}=C_{\text {aug }, u} \Delta \boldsymbol{x}_{\text {aug }}+D_{\text {aug }, u} \Delta \boldsymbol{u}
\end{array}\right.
$$


where $\quad \Delta \boldsymbol{x}_{\text {aug }}=\left[\begin{array}{c}\Delta \boldsymbol{x} \\ \Delta \boldsymbol{h}\end{array}\right], \quad A_{\text {aug }}=\left[\begin{array}{cc}A & L \\ 0 & 0\end{array}\right], \quad B_{\text {aug }}=\left[\begin{array}{c}B \\ 0\end{array}\right], \quad C_{\text {aug }, m}=\left[\begin{array}{ll}C_{m} & M_{m}\end{array}\right], \quad D_{\text {aug }, m}=D_{m}$, $C_{a u g, u}=\left[\begin{array}{ll}C_{u} & M_{u}\end{array}\right], \quad D_{\text {aug }, u}=D_{u}$ 。

After optimal estimating by Kalman filter, we can obtain

$$
\left\{\begin{array}{l}
\Delta \dot{\hat{\boldsymbol{x}}}_{\text {aug }}=A_{\text {aug }} \Delta \hat{\boldsymbol{x}}_{\text {aug }}+B_{\text {aug }} \Delta \boldsymbol{u}+K\left(\Delta \boldsymbol{y}_{m}-\Delta \hat{\boldsymbol{y}}_{m}\right) \\
\Delta \hat{\boldsymbol{y}}_{m}=C_{\text {aug }, m} \Delta \hat{\boldsymbol{x}}_{\text {aug }}+D_{\text {aug }, m} \Delta \boldsymbol{u} \\
\Delta \hat{\boldsymbol{y}}_{u}=C_{\text {aug }, u} \Delta \hat{\boldsymbol{x}}_{\text {aug }}+D_{\text {aug }, u} \Delta \boldsymbol{u}
\end{array}\right.
$$

where $K$ is Kalman gain matrix, $K=P C_{a u g, m}^{T} R^{-1}$, and $P$ can be obtained from Riccati equation,

$$
A_{\text {aug }} P+P A_{\text {aug }}^{T}-P C_{\text {aug }, m}^{T} R^{-1} C_{\text {aug }, m} P+Q=0
$$

In addition, the observability of $\left(A_{\text {aug }}, C_{\text {aug }, m}\right)$ should be checked for computing $K$.

Now, the outputs of NOBEM is used to the baseline of Kalman filter, which means the baseline in eq.(6) is $\left(\boldsymbol{x}_{\text {NOBEM }}, \boldsymbol{u}, \boldsymbol{y}_{\text {NOBEM }}, \boldsymbol{h}_{\text {NOBEM }}\right)$ instead of $\left(\boldsymbol{x}_{s s}, \boldsymbol{u}_{s s}, \boldsymbol{y}_{s s}, \boldsymbol{h}_{\text {ref }}\right)$,

$$
\left\{\begin{array}{l}
\dot{\hat{\boldsymbol{x}}}_{\text {aug }}=A_{\text {aug }}\left(\hat{\boldsymbol{x}}_{\text {aug }}-\boldsymbol{x}_{\text {aug }, N O B E M}\right)+K\left(\boldsymbol{y}_{m}-\hat{\boldsymbol{y}}_{m}\right) \\
\hat{\boldsymbol{y}}_{m}=C_{a u g, m}\left(\hat{\boldsymbol{x}}_{a u g}-\boldsymbol{x}_{\text {aug }, N O B E M}\right)+\boldsymbol{y}_{m, N O B E M} \\
\hat{\boldsymbol{y}}_{u}=C_{a u g, ~}\left(\hat{\boldsymbol{x}}_{\text {aug }}-\boldsymbol{x}_{\text {aug }, N O B E M}\right)+\boldsymbol{y}_{u, N O B E M}
\end{array}\right.
$$

Since the estimated values $\hat{\boldsymbol{h}}$ in eq.(8) is fed back to NOBEM, the updated model becomes $\left(\hat{\boldsymbol{x}}_{\text {NOBEM }}, \boldsymbol{u}, \hat{\boldsymbol{y}}_{\text {NOBEM }}, \hat{\boldsymbol{h}}_{\text {NOBEM }}\right)$. After combining these two parts,

$$
\left\{\begin{array}{l}
\dot{\hat{\boldsymbol{x}}}_{\text {aug }}=A_{\text {aug }}\left(\hat{\boldsymbol{x}}_{\text {aug }}-\hat{\boldsymbol{x}}_{\text {aug }, N O B E M}\right)+K\left(\boldsymbol{y}_{m}-\hat{\boldsymbol{y}}_{m}\right) \\
\hat{\boldsymbol{y}}_{m}=C_{a u g, m}\left(\hat{\boldsymbol{x}}_{a u g}-\hat{\boldsymbol{x}}_{a u g, N O B E M}\right)+\hat{\boldsymbol{y}}_{m, N O B E M} \\
\hat{\boldsymbol{y}}_{u}=C_{a u g, u}\left(\hat{\boldsymbol{x}}_{\text {aug }}-\hat{\boldsymbol{x}}_{a u g, N O B E M}\right)+\hat{\boldsymbol{y}}_{u, N O B E M}
\end{array}\right.
$$

The Eq.(9) is called IHKF and its inside structure is shown in Fig.3. Furthermore, the control inputs and matrices $B, D_{\text {aug }, m}, D_{\text {aug }, u}$ do not appear in this equation since the effect of control inputs is accounted for by the NOBEM.

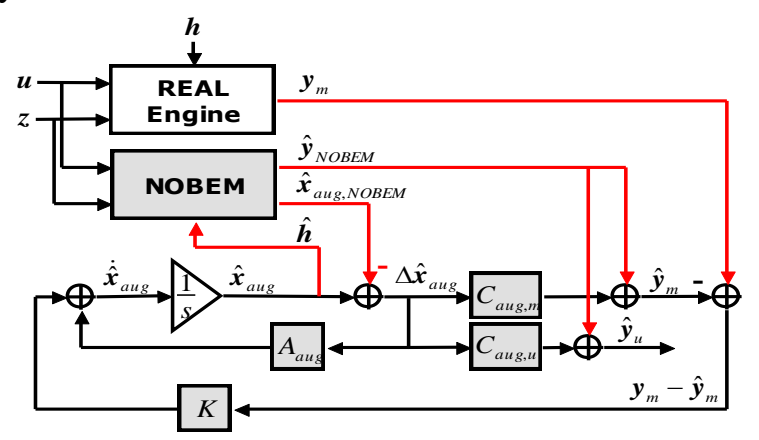

Fig. 3 The inside structure of IHKF

\subsubsection{PWKF}

At a flight condition, PWKF should be built since aircraft engine has a plurality of steadystate points such as idle, cruise and maximum. Due to the specialty of this hybrid structure, the baseline and some system matrixes need not to be computed, which simplifies the design processes. Furthermore, the linear part of eq.(8) only to be computed, 


$$
\left\{\begin{array}{l}
A_{\text {aug }}=A_{\text {aug }, s s}(\boldsymbol{\eta}) \\
C_{\text {aug }, m}=C_{a u g, m, s s}(\boldsymbol{\eta}) \\
C_{a u g, u}=C_{a u g, u, s s}(\boldsymbol{\eta}) \\
K=K_{s s}(\boldsymbol{\eta})
\end{array}\right.
$$

Where $\boldsymbol{\eta}$ is scheduling vector and subscript 'ss' denotes steady-state. So, PWKF shown in Fig.4 can be obtained via recalling this equation into eq.(9),

$$
\left\{\begin{array}{l}
\dot{\hat{\boldsymbol{x}}}_{\text {aug }}=A_{\text {aug }, \text { ss }}(\boldsymbol{\eta})\left(\hat{\boldsymbol{x}}_{\text {aug }}-\hat{\boldsymbol{x}}_{\text {aug }, N O B E M}\right)+K_{s s}(\boldsymbol{\eta})\left(\boldsymbol{y}_{m}-\hat{\boldsymbol{y}}_{m}\right) \\
\hat{\boldsymbol{y}}_{m}=C_{a u g, m, s s}(\boldsymbol{\eta})\left(\hat{\boldsymbol{x}}_{\text {aug }}-\hat{\boldsymbol{x}}_{\text {aug }, N O B E M}\right)+\hat{\boldsymbol{y}}_{m, N O B E M} \\
\hat{\boldsymbol{y}}_{u}=C_{a u g, u, s s}(\boldsymbol{\eta})\left(\hat{\boldsymbol{x}}_{\text {aug }}-\hat{\boldsymbol{x}}_{\text {aug }, N O B E M}\right)+\hat{\boldsymbol{y}}_{u, N O B E M}
\end{array}\right.
$$

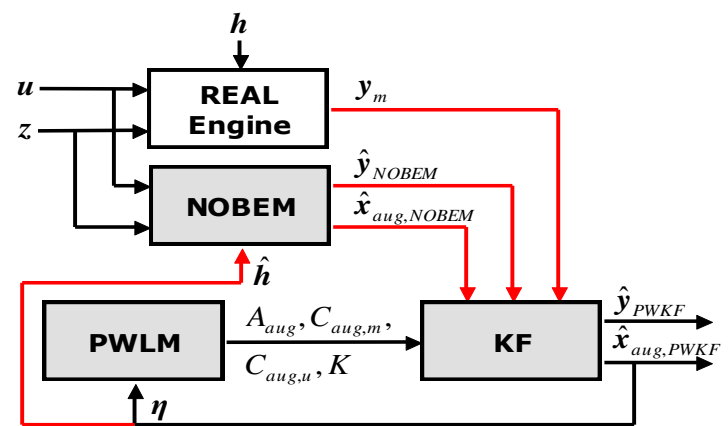

Fig. 4 The inside structure of PWKF

\subsubsection{Extend to the flight envelop}

Known formulae for reducing the engine parameters to the sea-level conditions are often used for calculating aircraft engine model parameters in a broad range of the external operating conditions. The formula operates with the inlet temperature $T_{i n}$ and pressure $P_{i n}$. Hence, IHKF can be defined in the corrected form as follow:

$$
\left\{\begin{array}{l}
\dot{\boldsymbol{x}}_{\text {aug }}^{c o r}=A_{\text {aug }, s s}^{c o r}\left(\boldsymbol{\eta}^{c o r}\right)\left(\hat{\boldsymbol{x}}_{\text {aug }}^{c o r}-\hat{\boldsymbol{x}}_{\text {aug }, N O B E M}^{c o r}\right)+K_{s s}^{c o r}\left(\boldsymbol{\eta}^{c o r}\right)\left(\boldsymbol{y}_{m}^{c o r}-\hat{\boldsymbol{y}}_{m}^{c o r}\right) \\
\hat{\boldsymbol{y}}_{m}^{c o r}=C_{a u g, m, s s}^{c o r}\left(\boldsymbol{\eta}^{c o r}\right)\left(\hat{\boldsymbol{x}}_{\text {aug }}^{c o r}-\hat{\boldsymbol{x}}_{\text {aug }, N O B E M}^{c o r}\right)+\hat{\boldsymbol{y}}_{m, N O B E M}^{c o r} \\
\hat{\boldsymbol{y}}_{u}^{c o r}=C_{a u g, u, s s}^{c o r}\left(\boldsymbol{\eta}^{c o r}\right)\left(\hat{\boldsymbol{x}}_{\text {aug }}^{c o r}-\hat{\boldsymbol{x}}_{a u g, N O B E M}^{c o r}\right)+\hat{\boldsymbol{y}}_{u, N O B E M}^{c o r}
\end{array}\right.
$$

where superscript 'cor' denotes corrected parameters $; \boldsymbol{\eta}^{\text {cor }}=f\left(\boldsymbol{x}^{\text {cor }}, \boldsymbol{u}^{\text {cor }}\right) ; A_{\text {aug,ss }}^{\text {cor }}=P_{\dot{x}_{a \mathrm{ag}}}^{-1} A_{\text {alg, ss }} P_{x_{\text {agg }}}$;

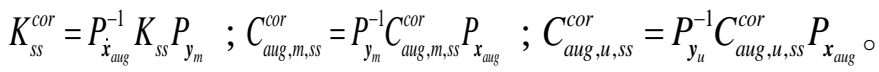

The diagonal matrix $P_{\dot{x}_{\text {aug }}}, P_{x_{\text {aug }}}, P_{\boldsymbol{y}_{m}}$ and $P_{\boldsymbol{y}_{u}}$ connect physical and corrected parameters:

$$
\dot{\boldsymbol{x}}_{\text {aug }}=P_{\dot{\boldsymbol{x}}_{\text {aug }}} \dot{\boldsymbol{x}}_{\text {aug }}^{\text {cor }}, \boldsymbol{x}_{\text {aug }}=P_{\boldsymbol{x}_{\text {aug }}} \boldsymbol{x}_{\text {aug }}^{\text {cor }}, \boldsymbol{y}_{m}=P_{\boldsymbol{y}_{m}} \boldsymbol{y}_{m}^{\text {cor }}, \boldsymbol{y}_{u}=P_{\boldsymbol{y}_{u}} \boldsymbol{y}_{u}^{\text {cor }}
$$

\section{Simulation}

Taken a military turbofan engine as an example, an IHKF from idle to maximum was built.

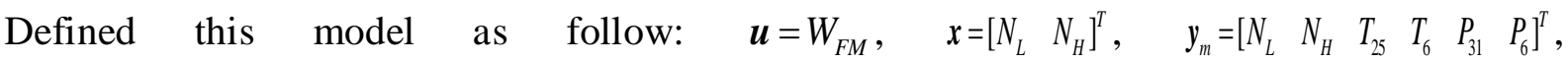
$\boldsymbol{y}_{u}=\left[\begin{array}{llll}F_{N} & S_{M F} & W_{21} & T_{41}\end{array}\right]^{T}, \boldsymbol{h}=\left[\begin{array}{llll}D_{E L} & D_{E H} & D_{W L} & D_{W H}\end{array}\right]^{T}, z=\left[\begin{array}{ll}T_{1} & P_{1}\end{array}\right]^{T}, \boldsymbol{\eta}=W_{F M}$. With regard to measured noise, the actual sensor standard deviations are probably between $0.1 \%$ and $1 \%$ in percent of steady-state values at ground maximum power condition. For simplicity, we assume them to be $0.2 \%$ here. 
For verifying the functionality of this model, a Simulink model with FADEC based on Fig.1 was built and taken a series of simulation examples such as steady-state mode, transitionstate/abnormal mode and compared with THKF in synthetic mode. In this Simulink model, the real engine was replaced by the aero-thermodynamic component-level model of this engine and the FADEC was built by the actual control logic and LQG/LTR method.

\subsection{Steady-State Mode}

When the PDF are changed respectively by $4 \%, 3 \%, 2 \%, 1 \%$ at $\mathrm{t}=5 \mathrm{~s}, 25 \mathrm{~s}, 45 \mathrm{~s}, 65 \mathrm{~s}$ and $5 \mathrm{~s}, 5 \mathrm{~s}$, $45 \mathrm{~s}, 45 \mathrm{~s}$ at ground maximum power condition, the IHKF can recognize their changes as shown in Fig.5. For the fan efficiency $D_{E L}$, IHKF can recognize rapidly its changes when it drops $4 \%$ at $\mathrm{t}=5 \mathrm{~s}$, as shown in Fig.5(a). The compressor flow capacity $D_{w H}$ is changed apparently, but the other parameters are slightly changed in transition, but go to zero rapidly. The PDF estimation is successfully completed in 8s. The results of other PDF changes are similar. For simultaneous PDF changes, the IHKF also can complete their estimations as shown in Fig.5(b).

Under the successful estimation of PDF, taken Fig.5(a) as an example, the outputs of this model can be seen in Fig.5(c)(d). For the limited space, we just list the fan speed and net thrust which respectively denotes the measured outputs and unmeasured outputs. From these figures, the outputs of NOBEM and PWKF match with the real engine after 8s transition, almost no steady-state deviation. Moreover, compared with the outputs disturbance condition of PWKF, that of NOBEM is more effective. In addition, a lot of simulations at different flight conditions and power conditions show the results are similar.

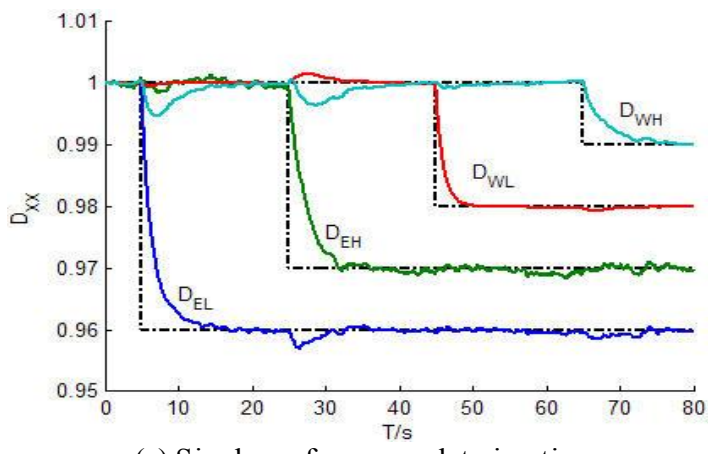

(a) Single performance deterioration

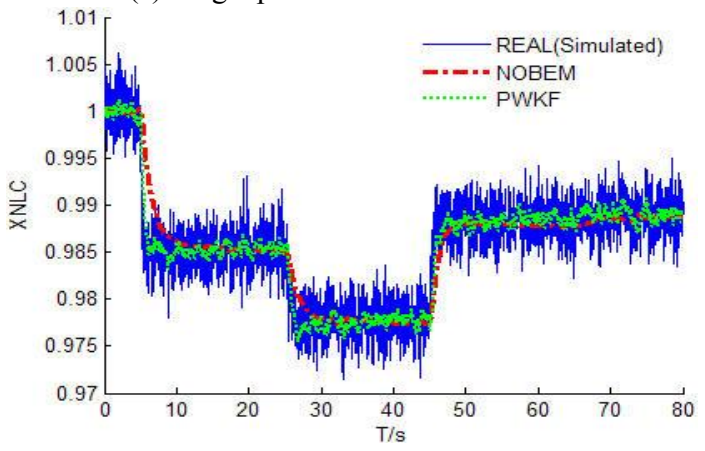

(c) Fan speed

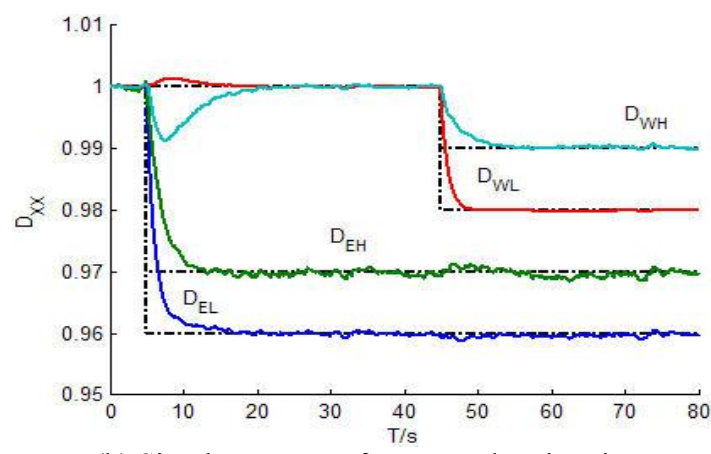

(b) Simultaneous performance deterioration

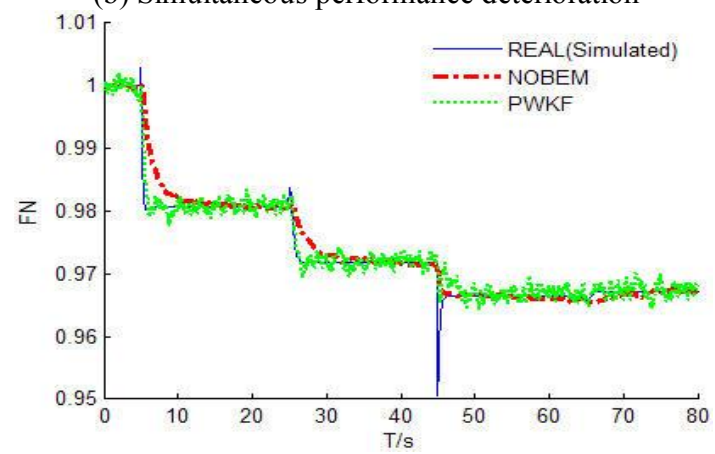

(d) Net thrust

Fig.5 Simulation results at the steady-state mode 


\subsection{Transition-State/Abnormal Mode}

During switching from steady-state mode to transition-state mode, two possible conditions exist, including completed accurate estimation and not, which have an influence on the model estimation precision at transition-state mode. Although the latter condition can be ignored in the real world because of the small probability of happen, the results are similar to that at abnormal mode.

At ground condition, we took the close-loop simulation by PLA changes shown in Fig.6(a), where two efficiency factors and two flow efficiency factors are dropped respectively by $4 \%$, $3 \%$ and $2 \%, 1 \%$ at $19.5 \mathrm{~s}$ and $45 \mathrm{~s}$. The results are shown in Fig.6(b)-(d).

Seen from red dashed frame in Fig.6(b), the IHKF is in the state of uncompleted accurate estimation and has big deviation from the real performance deterioration. Fig.6(c) shows the steady-state outputs of NOBEM and PWKF exist a little deviation simultaneously, and the former's amplitude is bigger than the latter's. Accordingly, seen from blue dashed frame in Fig.6(b), the IHKF is in the state of completed accurate estimation and has small deviation form the real performance deterioration. Fig.6(d) shows the steady-state outputs of NOBEM and PWKF do not exist deviation, but the latter does in transition since this linear model can't describe the nonlinear characteristics of aircraft engine.

So the results of simulation shows that the outputs of NOBEM are more effective in the state of completed accurate estimation while the outputs of PWKF are more effective in the state of uncompleted accurate estimation, and also verify the functionality of IHKF at abnormal mode.
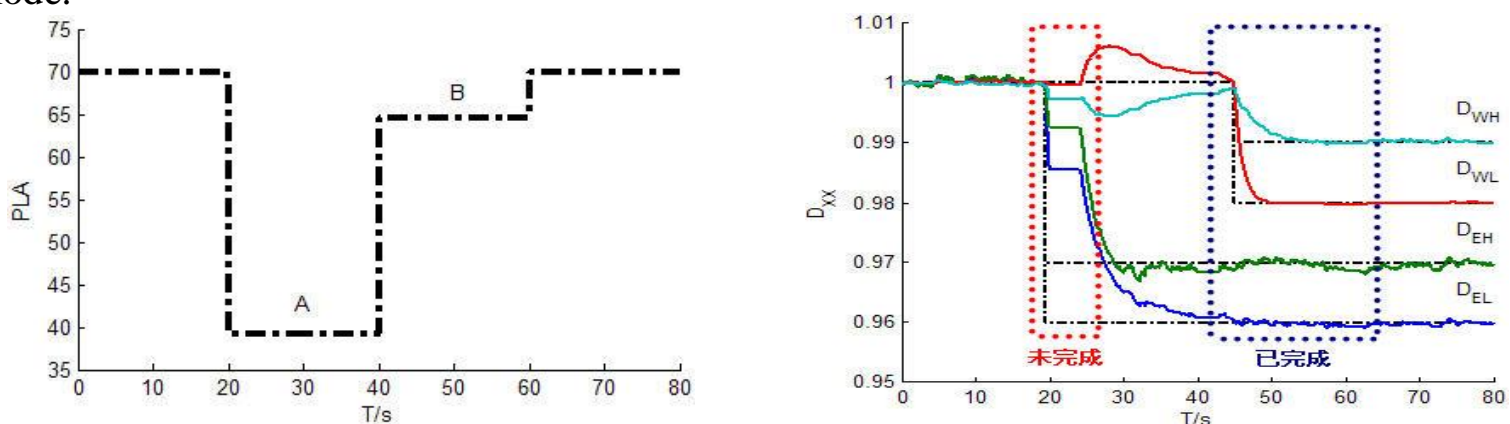

(a) PLA

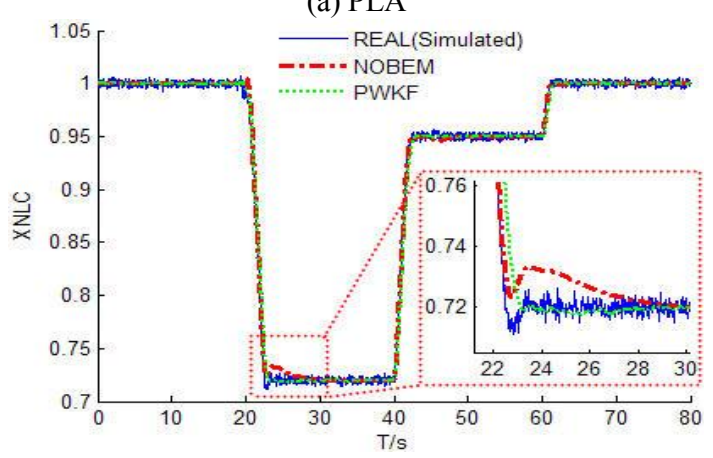

(b) Performance deterioration estimation

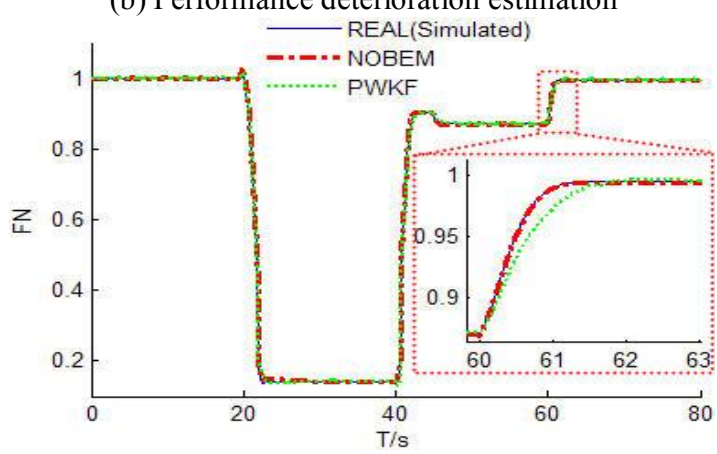

(c) Fan speed

(d) Net thrust

Fig. 6 Simulation results at the transition-state/abnormal-state mode

\subsection{Compare in Synthetic Mode}

For investigating the effectiveness of IHKF at FOD, compared with THKF, a standard work cycle was simulated. Where the curve of $H, M a$ and PLA are shown in Fig.(a); the PDF is linear against time with the amplitude of 4\%,3\%,2\%, 1\%; the FOD happens at $\mathrm{t}=500 \mathrm{~s}$ and all PDF drop 1\%. The results of simulation are shown in Fig.7(b)-(d). 
Seen from Fig.7(b), the PDF of THKF in dashed are constant while that of IHKF successfully estimate the real performance deterioration, including the step changes at $t=500 \mathrm{~s}$. Accordingly, with regard to the outputs as shown in Fig.7(c)(d), both of them match with the real engine before $t=500$ s while the IHKF does after $t=500 \mathrm{~s}$, but the THKF not.

Therefore, the results of simulation show that THKF can match with the real engine under the update period at the slight performance deterioration condition because it possesses inherent robustness to uncertainty, but not at the severe condition. By contraries, the IHKF possesses better adaptivity to FOD.
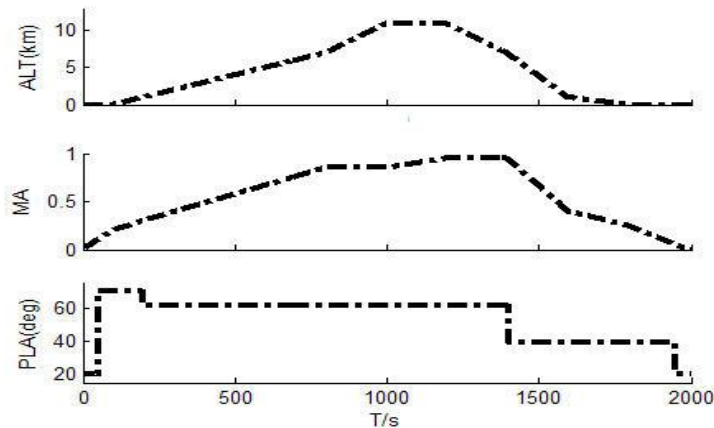

(a) The curve of ALT, Ma and PLA

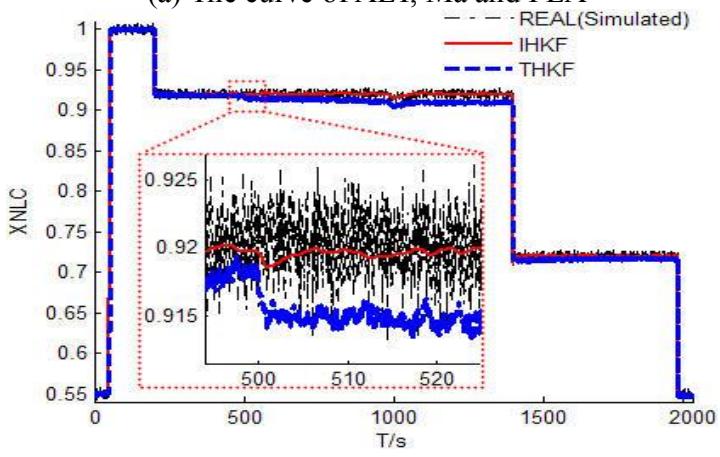

(c) Fan speed

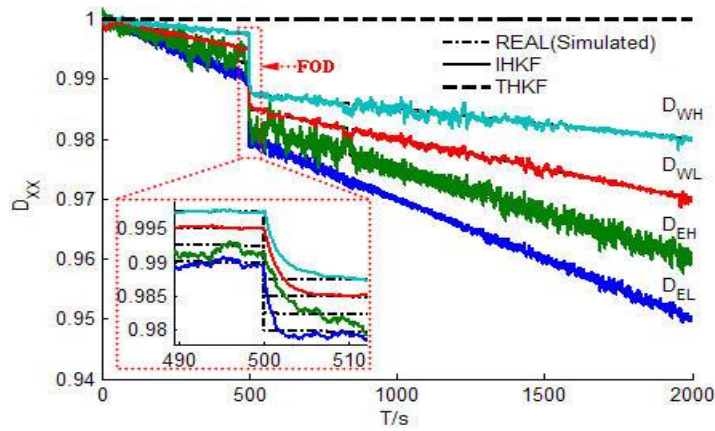

(b) Performance deterioration estimation

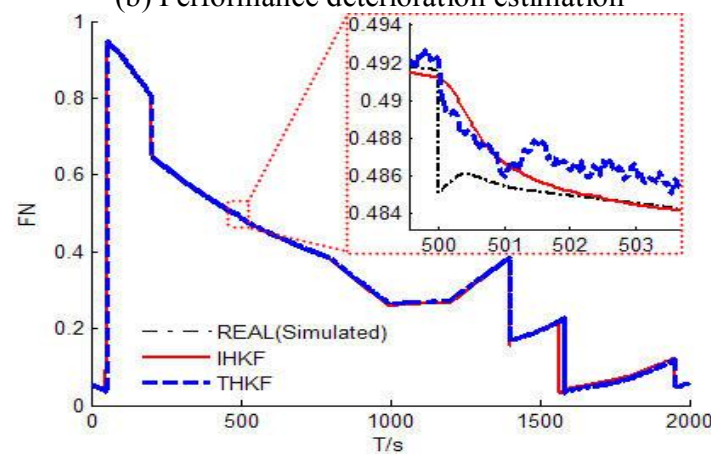

(d) Net thrust

Fig. 7 Compared simulation results at the several state modes

\section{Conclusion}

This paper proposes an IHKF for in-flight aircraft engine performance estimation and describes model structure, work mode and the key techniques of model design in details. By this approach applied to a turbofan engine, we took a series of simulation examples such as steady-state mode, transition-state/abnormal mode and compared with THKF in synthetic mode. The results of these simulations show that the model can estimate the real engine performance effectively in the whole flight envelope, different engine states and severe performance deterioration condition, which lays the foundation for IEC, PSC and FDIA.

\section{References}

[1] Luppold R H, Roman J R, Gallops G W, et al. Estimating in-flight engine performance variations using kalman filter concepts[R]. AIAA, ASME, SAE, and ASEE, Joint Propulsion Conference, 25th, Monterey, CA, July 10-13, 1989. 10 p., 1989.

[2] Brotherton T, Volponi A, Luppold R, et al. Estorm:enhanced self tuning on-board realtime engine model[C]. Big Sky MT, United states: Proceedings of the 2003 IEEE Aerospace Conference, 2003.

[3] Simon D, Simon D L. Kalman filtering with inequality constraints for turbofan engine health estimation[R]. NASA/TM-2003-212111, 2003. 
[4] Xinde Wang, Jianguo Sun, Songlin Li. Application of Neural Networks to Areoengine Adaptive Modeling[J]. Journal of Aerospace Power. 2003, 18(06): 845-849.

[5] Volponi A, Brotherton T. A bootstrap data methodology for sequential hybrid engine model building[C]. 2005. 3463-3471.

[6] Borguet S, Dewallef P, Leonard O. A way to deal with model-plant mismatch for a reliable diagnosis in transient operation[C]. Barcelona, Spain: 2006.

[7] Chunfei Yuan, Hua Yao, Gang Yang. On-board Real-time Adaptive Model of Aeroengine[J]. Acta Aeronautica et Astronautica Sinica. 2006, 27(4): 561-564.

[8] Volponi A, Brotherton T, Luppold R. Empirical tuning of an on-board gas turbine engine model for real-time module performance estimation[J]. Journal of Engineering for Gas Turbines and Power. 2008, 130(2): 21604.

[9] Weibin Huang, Jinquan Huang. On board self-tuning model for aero-engine fault diagnostics[J]. Journal of Aerospace Power. 2008, 23(3): 580-584.

[10] Xianquan Zou, Jianguo Sun, Haibo Zhang. Adaptive model of rotor/ turbo-shaft engine[J]. Journal of Beijing University of Aeronautics and Astronautics. 2009, 35(10): 1192-1196.

[11] Sugiyama N. System identification of jet engines[J]. Journal of Engineering for Gas Turbines and Power. 2000, 122(1): 19-26.

[12] Kobayashi T, Simon D L. Integration of on-line and off-line diagnostic algorithms for aircraft engine health management $[\mathrm{J}]$. Journal of Engineering for Gas Turbines and Power. 2007, 129(4): 986-993.

[13] Kumar A, Viassolo D. Model-based fault tolerant control[R]. NASA/CR-2008-215273, 2008.

[14] Tagashira T, Mizuno T, Koh M, et al. Atf test evaluation of model based control for a single spool turbojet engine[C]. 2009. 673-685.

[15] Sugiyama N. Derivation of abcd system matrices from nonlinear dynamic simulation of jet engines[R]. AIAA-92-23319, 1992.

[16] Zhengping Feng, Jianguo Sun. A new method for establishing a state space model of aeroengine[J]. Journal of Aerospace Power. 1998, 13(4): 435-438. 